\title{
Um pouco de complexidade na Linguística Aplicada
}

\author{
Claudio de Paiva Franco \\ Universidade Federal do Rio de Janeiro \\ cpaivafranco@yahoo.com.br
}

\section{Resumo}

Este trabalho tem por objetivo discutir algumas possibilidades de aplicação do paradigma da complexidade no campo da Linguística Aplicada. Para isso, pretendo apresentar as características e os comportamentos dos sistemas complexos com base em uma revisão de literatura sobre a teoria da complexidade. Além disso, compartilho os resultados de alguns estudos fundamentados na teoria da complexidade desenvolvidos em um Programa de Pós-Graduação em Estudos Linguísticos (Linguística Aplicada).

Palavras-chave: Complexidade. Sistemas complexos. Linguística Aplicada.

\begin{abstract}
This paper aims to discuss some application possibilities of the paradigm of complexity in the field of Applied Linguistics. In this paper I intend to present the characteristics and behaviors of complex systems based on a review of literature on complexity theory. Besides, I also share the results of some studies grounded in complexity theory that were developed in a Graduate Program in Linguistic Studies (Applied Linguistics).
\end{abstract}

Keywords: Complexity. Complex systems. Applied Linguistics.

\section{Introdução}

O paradigma da complexidade permitiu que diversos campos do saber reexaminassem seus construtos, distanciando-se da visão fragmentada, linear e de simplificação do pensamento pertencente ao racionalismo newtoniano. A complexidade inaugura novas formas de conceber o universo à luz da desordem e da incerteza. Trata-se de uma noção mais abrangente de compreender fenômenos complexos que não podem ser descritos sob a lógica reducionista. Essa mudança de paradigma, que começou com as ciências físicas e biológicas, é 
também encontrada em pesquisas nas mais diversas áreas, como a Linguística Aplicada.

Larsen-Freeman (1997) foi a precursora a adotar a teoria do caos/complexidade na Linguística Aplicada. Seu artigo seminal "Chaos/complexity science and second language acquisition", publicado na Applied Linguistics em 1997, tornou-se uma referência para estudiosos da área. A autora buscou estabelecer semelhanças entre as características dos sistemas complexos e da aquisição de segunda língua (ASL). Ela aprofundou seus estudos e publicou diversos trabalhos sobre a ASL à luz da complexidade (LARSEN-FREEMAN, 2000, 2002, 2006, 2009). Em 2008, Larsen-Freeman e Cameron publicaram o livro Complex systems and Applied Linguistics, no qual a teoria da complexidade é introduzida como uma metáfora para sistemas na Linguística Aplicada. Para as autoras (2008, p. 226), quando entendemos um sistema aplicado como complexo, adaptável e dinâmico, novas formas de conceituar propriedades e atividades são geradas. Além disso, consideramos novas questões sobre como as pessoas usam, aprendem e ensinam línguas, o que, por sua vez, exige novas formas de investigação.

Com a ampla repercussão dos estudos sobre complexidade na Linguística Aplicada, o periódico Applied Linguistics dedicou uma edição especial, em 2006, a trabalhos que tratavam da perspectiva complexa de entender a linguagem como um sistema complexo. Segundo essa perspectiva, os signos linguísticos são "produtos contextualizados da integração de diversas atividades por (determinados) indivíduos em determinadas situações comunicativas" (LARSEN-FREEMAN, 2006, p. 594).

Um número crescente de pesquisadores brasileiros da Linguística Aplicada (AUGUSTO, 2009; BRAGA, 2007, FRANCO, 2011, 2013; LEFFA, 2006, 2009; MARTINS, 2008; PAIVA, 2002, 2005a, 2005b, 2006, 2008, 2009, 2011; PARREIRAS, 2005; SADE, 2009; SILVA, 2008; SOUZA, 2011; TEIXEIRA, 2012; VETROMILLE-CASTRO, 2007)adota a complexidade como alternativa para investigar fenômenos complexos na Linguística Aplicada, como veremos mais adiante. Antes disso, faço uma breve apresentação, na próxima seção, das características e dos comportamentos dos sistemas complexos para que possamos compreender melhor o paradigma da complexidade. 


\section{Sistemas complexos}

A teoria da complexidade é também conhecida como a teoria dos sistemas complexos. De acordo com o New England Complex Systems Institute (NECSI), sistemas complexos constituem "um novo campo da ciência que estuda como as partes de um sistema concebem comportamentos do sistema como um todo e como esse sistema interage com seu ambiente". Para Waldrop (1992, p.11), o comportamento dos sistemas complexos emerge das interações entre seus componentes e não é descritível por uma única regra. São sistemas que exibem características inesperadas. Nas palavras de Paiva (2006, p. 91), "um sistema complexo não é um estado, mas um processo. Cada componente do sistema pertence a um ambiente construído pela interação entre suas partes". Alguns exemplos de sistemas complexos conhecidos são o clima, a fauna e a flora em um ecossistema, a dinâmica da transmissão de doenças infectocontagiosas e o funcionamento de células no corpo humano (LARSENFREEMAN; CAMERON, 2008).

Richardson, Cilliers e Lissack (2001, p. 7) afirmam que um sistema complexo pode ser descrito apenas como contendo um grande número de elementos com alto nível de interatividade, em que "a natureza dessa interatividade é essencialmente não-linear, contendo manifestações contínuas de feedback". Joslyn e Rocha (2000, p. 2) oferecem uma definição parecida e acrescentam que esse tipo de sistema apresenta, normalmente, "auto-organização hierárquica sob pressões seletivas".

Johnson (2007) afirma que um sistema com apenas dois constituintes é simples, mas quando há um terceiro elemento, tem-se uma multidão. Ao explorar a metáfora, o autor $(2007$, p. 4) explica que a multidão é um exemplo perfeito de fenômeno emergente, uma vez que é um fenômeno que emerge de um conjunto de pessoas em interação. Johnson (2007, p. 13-15) acredita que os sistemas complexos devem ter todas ou a maioria das seguintes características:

um conjunto de muitos objetos ou "agentes" em interação. A interação entre os agentes ocorre devido à proximidade física ou pelo fato de compartilharem uma informação em comum; 
- o comportamento desses objetos é afetado pela memória ou feedback. Um acontecimento passado pode influenciar um evento no presente ou ainda um evento pode interferir em outro evento simultâneo em local diferente;

- os objetos podem adaptar suas estratégias de acordo com seu histórico. A possibilidade de auto-organização permite que o agente adapte seu comportamento na esperança de melhorar seu desempenho;

- o sistema é, em geral, aberto. O sistema pode ser afetado pelo mundo externo. Sistemas fechados, ou seja, aqueles totalmente isolados do meio externo, são raros;

- o sistema parece ser vivo. Ele é movido por agentes que interagem entre si e se adaptam sob a influência de feedback;

o sistema exibe fenômenos emergentes geralmente surpreendentes que podem ser extremos. Como os sistemas estão longe do equilíbrio, fenômenos inesperados podem acontecer. $\mathrm{O}$ elemento surpresa ocorre pelo fato de não ser possível prever o que vai acontecer com o sistema ao se considerar apenas o conhecimento das propriedades de cada agente isoladamente;

- os fenômenos emergentes surgem, em geral, na ausência de qualquer tipo de "mão invisível" ou regulador central. Um sistema complexo pode se complexificar por si só, nunca sendo equivalente à soma de suas partes;

- o sistema exibe uma mistura complicada de comportamento ordenado e desordenado. Sistemas complexos transitam entre a ordem e a desordem.

Segundo Larsen-Freeman e Cameron (2008), a escola é um exemplo de sistema adaptativo complexo. Nas palavras das autoras (2008, p. 33), a escola é "um sistema aberto, e pode ser também uma estrutura dissipativa longe do equilíbrio, em que a ordem ou estabilidade dinâmica é, felizmente, a vivência pelos alunos da educação significativa e de afirmação da vida". Nesse sistema, encontramos componentes como professores, coordenadores pedagógicos, alunos, currículo, material didático, além de diversos 
fatores externos que podem influenciar esses componentes e interações.

Na próxima seção, compartilho alguns estudos (teses, artigos, livros e capítulos de livros) em Linguística Aplicada fundamentados na teoria da complexidade e desenvolvidos por pesquisadores brasileiros.

\section{Estudos à luz da complexidade em um programa de pós- graduação em Estudos Linguísticos}

Como já mencionado na Introdução deste artigo, um número crescente de pesquisadores no Brasil tem se dedicado a investigar questões da Linguística Aplicada à luz da complexidade. Este trabalho, no entanto, reúne apenas os estudos desenvolvidos no âmbito do Programa de Pós-Graduação em Estudos Linguísticos da Universidade Federal de Minas Gerais, mais especificamente as teses defendidas na área de Linguística Aplicada. A determinação desse escopo se deve à minha participação no grupo de pesquisa sobre complexidade, durante a fase de doutoramento na UFMG, coordenado pela professora Vera Menezes, e ao fato de a UFMG ter sido a instituição com o maior número de teses defendidas que contemplam a complexidade.

Cabe destacar, neste trabalho, a importância da UFMG nos estudos sobre complexidade desenvolvidos no Brasil. Como a complexidade é um paradigma emergente, ainda há poucos trabalhos de mestrado e doutorado que investigam fenômenos a partir dessa visão. Além disso, no campo da Linguística Aplicada, há poucos pesquisadores brasileiros que trabalham com a complexidade em alguma linha de pesquisa na pós-graduação. A considerar o número de orientações concluídas na última década (2005-2014), destacam-se os seguintes pesquisadores: Paiva (UFMG) com dez teses; Freire (PUC$\mathrm{SP}$ ) com seis teses e três dissertações; e Vetromille-Castro (UFPEL), com quatro dissertações.

Desde a publicação de seu memorial "Caleidoscópio: fractais de uma oficina de ensino aprendizagem", a pesquisadora Vera Menezes tem se dedicado à publicação de diversos de trabalhos que tratam do paradigma da complexidade. Paiva e Nascimento (2009) organizaram o livro Sistemas adaptativos complexos: lingua(gem) e aprendizagem, uma coletânea composta de 12 capítulos dedicados à 


\section{Um pouco de complexidade}

discussão de assuntos relacionadost anto à Linguística quanto à Linguística Aplicada sob as lentes da complexidade. Alguns capítulos abordam temas como ASL, formação de professores de língua estrangeira, processos de interação, comunidades de aprendizagem onlinea partir de um olhar multidimensional e dinâmico.

No Quadro 1, apresento os títulos das teses fundamentadas na perspectiva da complexidade que foram defendidas na UFMG, na área de Linguística Aplicada, organizadas por data de publicação.Em seguida,faço um breve resumo de cada uma.

A primeira tese orientada pela perspectiva teórica dos sistemas complexos foi defendida em 2005 por Parreiras. Em seu trabalho, o autor buscou investigar a sala de aula digital a partir da observação dos fluxos interacionais ocorridos entre os alunos de duas turmas simultâneas oferecidas a distância. Os resultados da pesquisa apontam para a confirmação da hipótese de que os fluxos das interações ocorridas nos dois ambientes digitais observados os caracterizam como sistemas complexos.

$\mathrm{Na}$ segunda tese, Braga (2007) buscou uma melhor compreensão dos fatores que podem contribuir favoravelmente para a construção de significado compartilhado em contextos colaborativos via internet. Além disso, a autora buscou algumas evidências empíricas de propriedades dos sistemas complexos na experiência colaborativa on-line e também padrões emergentes, fatores e componentes que podem influenciar positiva ou negativamente a colaboração e a construção de sentidos nesse contexto. Braga demonstrou que das interações entre os pares das comunidades autônomas de aprendizagem on-line emergem padrões de dimensões sociais, cognitivas, instrucionais e gerenciais, tais como a reciprocidade, a construção compartilhada de significado, a liderança distribuída, bem como o caráter fractal das comunidades de um curso on-line.

Em 2008, Martins concluiu sua pesquisa, tendo investigado as dinâmicas interativas ocorridas em uma disciplina de escrita em inglês como língua estrangeira oferecida na modalidade semipresencial, ou seja, com aulas face a face e on-line. A tese do autor é sustentada em uma perspectiva ecossistêmica, incluindo pressupostos da teoria da complexidade e de abordagens ecológicas às relações de ensinoaprendizagem de línguas. Os resultados ressaltam a importância de desenhos instrucionais mistos que busquem a convergência das 
modalidades de ensino presencial e on-line, explorando as potencialidades de cada modalidade.

Quadro 1. Teses defendidas sobre complexidade no PPGLE da UFMG ${ }^{l}$

\begin{tabular}{|l|l|l|}
\hline Título & Autor & $\begin{array}{l}\text { Ano de } \\
\text { defesa }\end{array}$ \\
\hline $\begin{array}{l}\text { A sala de aula digital sob a perspectiva dos sistemas } \\
\text { complexos: uma abordagem qualitativa }\end{array}$ & Parreiras & 2005 \\
\hline $\begin{array}{l}\text { Comunidades autônomas de aprendizagem on-line na } \\
\text { perspectiva da complexidade }\end{array}$ & Braga & 2007 \\
\hline $\begin{array}{l}\text { A emergência de eventos complexos em aulas on-line e } \\
\text { faceaface: uma abordagem ecológica }\end{array}$ & Martins & 2008 \\
\hline $\begin{array}{l}\text { A dinâmica caleidoscópica do processo de } \\
\text { aprendizagem colaborativa: um estudo na perspectiva } \\
\text { da complexidade/caos }\end{array}$ & Silva & 2008 \\
\hline $\begin{array}{l}\text { Identidade e aprendizagem de inglês sob a ótica do } \\
\text { caos e dos sistemas complexos }\end{array}$ & Sade & 2009 \\
\hline $\begin{array}{l}\text { O processo de desenvolvimento da competência } \\
\text { linguística em inglês na perspectiva da complexidade }\end{array}$ & Augusto & 2009 \\
\hline $\begin{array}{l}\text { Dinamicidade e adaptabilidade em comunidades } \\
\text { virtuais de aprendizagem: uma textografia à luz do } \\
\text { paradigma da complexidade }\end{array}$ & Souza & 2011 \\
\hline $\begin{array}{l}\text { Difusão tecnológica no ensino de línguas: } \text { o uso de } \\
\text { computadores portáteis nas aulas de Língua } \\
\text { Portuguesa sob a ótica da complexidade }\end{array}$ & Teixeira & 2012 \\
\hline $\begin{array}{l}\text { Autonomia na aprendizagem de inglês: um estudo com } \\
\text { nativos digitais sob as lentes do caos e da } \\
\text { complexidade }\end{array}$ & Franco & 2013 \\
\hline $\begin{array}{l}\text { Estágio supervisionado com uso de ambientes } \\
\text { virtuais: possibilidades colaborativas }\end{array}$ & Silva & 2013 \\
\hline
\end{tabular}

Ainda no mesmo ano, à luz dos princípios teóricos dos sistemas complexos, Silva (2008) analisou 1.370 e-mails trocados entre aprendizes adultos em uma lista de discussão. Silva pretendeu mostrar que o processo de colaboração para a aprendizagem é uma propriedade natural nessa comunidade. Os resultados revelam que a

${ }^{1}$ Todas as teses mencionadas neste quadro podem ser encontradas em:

<http://www.letras.ufmg.br/poslin/tese.asp>. 


\section{Um pouco de complexidade}

colaboração foi considerada um evento dinâmico que emergiu no interstício entre o previsível e o imprevisível do sistema. Por fim, Silva concluiu que o andaime instrucional, quando focado sob as lentes do caos/complexidade, é uma metáfora que se apresenta inadequada para representar a dinâmica complexa da aprendizagem colaborativa.

No ano seguinte, aliada à teoria do caos/complexidade, Sade (2009) apresentou a identidade social no panorama contemporâneo como um sistema caótico e complexo. Demonstrou ainda, em sua pesquisa-narrativa, a inter-relação existente entre os processos de (re)construção identitária e o de aprendizagem de língua estrangeira. Os resultados indicam que os processos de reconstrução identitária e de aprendizagem de línguas guardam entre si uma relação de interação e de inteiração. A pesquisadora ainda sugeriu o emprego de um novo termo - identidades fractalizadas - para se referir à complexificação dafractalização do sistema identitário do ser humano.

Outra tese à luz da perspectiva da complexidade também foi defendida em 2009. Trata-se de um estudo de caso do tipo etnográfico. Augusto (2009) objetivou buscar uma melhor compreensão das características e do funcionamento do processo de desenvolvimento da competência linguística de uma professora de língua inglesa participante de um curso de educação continuada. Os resultados revelam que o desenvolvimento da competência linguística, sob uma perspectiva emergentista, é um processo não linear e imprevisível que ocorre por meio da inter-relação de vários fatores em diferentes estágios e escalas de tempo.

Souza (2011) argumentou que os ambientes virtuais de aprendizagem (AVA) devem ser considerados comunidades virtuais de aprendizagem que emergem das ações dos agentes no ambiente. A autora investigou dois AVAs, definindo-os como sistemas compostos por gêneros digitais e partindo do pressuposto de que um AVA é um sistema adaptativo complexo. As análises revelam que a produção textual das comunidades discursivas emergentes nos AVAs foi influenciada pelo acoplamento aninhado de camadas distintas do suporte digital, dos gêneros textuais e dos propósitos comunicativos e, ainda, pelos propiciamentos (affordances) percebidos e efetivados por professoras e aprendizes. Souza conclui que os AVAs oferecem oportunidades para uma experiência pedagógica que deve ter como foco propiciamentos significativos para que as tarefas que propiciam a 
dinamicidade do AVA tendam a assegurar dinâmicas pertinentes para a formação almejada.

Teixeira (2012), orientada pelo paradigma da complexidade, buscou apresentar o modo como os docentes de Língua Portuguesa vêm incorporando laptops educacionais em suas aulas. Na referida pesquisa de cunho qualitativo foram analisadas, por meio de narrativas e depoimentos, as percepções dos participantes (docentes e discentes) do programa Um Computador por Aluno (UCA), que oferece laptops para toda a rede pública do município de Tiradentes, em Minas Gerais. Os resultados direcionam para as implicações da inserção da tecnologia nas aulas de Língua Portuguesa, e os dados refletem as dificuldades e os receios enfrentados pelos professores durante a implantação do Projeto UCA.

Franco (2013) apresentou um estudo de caso que tem como principal objetivo conhecer as experiências de aprendizagem, mais especificamente o desenvolvimento da autonomia na aprendizagem de inglês de um grupo de alunos do ensino médio de uma instituição escolar federal localizada no Rio de Janeiro. Com base em narrativas multimídia de aprendizagem desse grupo de alunos nativos digitais, o autor explorou, à luz da teoria do caos/complexidade, possíveis relações entre atratores (padrões de comportamento) e autonomia de nativos digitais no contexto de aprendizagem de inglês. Os resultados gerados revelam que: (1) todas as evidências de desenvolvimento da autonomia emergiram de um atrator (práticas pedagógicas avaliadas negativamente, práticas pedagógicas avaliadas positivamente e práticas socioculturais extraclasse); (2) as novas tecnologias ou os propiciamentos de uso das novas tecnologias por si só não promoveram o desenvolvimento da autonomia; (3) somente os atratores que convergiram para uma atitude positiva à língua estrangeira puderam promover o desenvolvimento da autonomia; (4) o desenvolvimento da autonomia, nos sistemas com atratores que convergiram para uma atitude negativa à língua estrangeira, somente emergiu quando houve um atrator que convergiu para uma atitude positiva à língua estrangeira; (5) o desenvolvimento da autonomia de nativos digitais emergiu, total ou parcialmente, em contextos informais de aprendizagem ricos em propiciamentos, como, por exemplo, na internet. 
Por fim, Silva (2013) adotou a perspectiva da complexidade e da aprendizagem situada para a análise dos dados em sua tese. Os resultados da pesquisa revelam que o meio virtual é um aliado pedagógico à intervenção complexa que é a formação de professores. Segundo a pesquisadora, interação, imersão virtual, aprendizagem colaborativa, construção coletiva e reflexão são aspectos importantes que devem ser levados em consideração na formação docente atual, para que haja uma abertura à reestruturação da experiência pedagógica.

\section{Considerações finais}

Neste trabalho, discuti as características e os comportamentos dos sistemas complexos e apresentei os estudos norteados pelo paradigma da complexidade desenvolvidos no âmbito do Programa de Pós-Graduação em Estudos Linguísticos da Universidade Federal de Minas Gerais, mais especificamente na área de Linguística Aplicada. Com base em uma breve revisão de literatura sobre complexidade, descobri que a concepção de complexidade transcende a definição de elementos entrelaçados, que formam um tecido em conjunto. $\mathrm{O}$ termo refere-se a múltiplas interações e traz em seu bojo a noção de imprevisibilidade. À medida que percebemos que as interações não cessam, afastamo-nos cada vez mais da ideia de completude e de certezas inquestionáveis.

Com base no entendimento dos sistemas complexos e de suas características, atentei para a dinâmica que subjaz à complexidade. Vi que esses sistemas são formados por elementos ou agentes que interagem entre si ao longo do tempo e de formas distintas. A troca de energia entre as partes e com o meio externo permite que os sistemas se auto-organizem de modo que o todo se torna mais complexo. Nas palavras de Johnson (2007, p. 17), a complexidade é uma visão de mundo que "dá um tapa na cara das abordagens tradicionais reducionistas". Adotar a epistemologia da complexidade implica comprometer-se em rejeitar a visão que teima em dissolver as relações, simplificar o complexo e separar o que está entrelaçado.

Os pesquisadores que submetem o olhar à complexidade não embarcam em uma tarefa fácil. Seria mais cômodo continuar a enxergar as relações e os fenômenos complexos de forma linear e 
esgotar as possibilidades de resposta para nossos questionamentos. À medida que complexificamos, damo-nos conta de que as interações não cessam e cada vez mais nos afastamos da ideia de completude. Adotar a epistemologia da complexidade é uma escolha, antes de tudo, pessoal. É o comprometer-se em problematizar constantemente e querer viver no limite do caos, pois é lá que o turbilhão de interações nos pode renovar e abalar nossas quase-verdades, que, em breve, transformar-se-ão em verdadeiras incertezas.

\section{Referências}

AUGUSTO, Rita C. O processo de desenvolvimento da competência linguística em inglês na perspectiva da complexidade. 2009. Tese (Doutorado em Estudos Linguísticos) - Faculdade de Letras, Universidade Federal de Minas Gerais, Belo Horizonte, 2009.

BRAGA, Junia. C. F. Comunidades autônomas de aprendizagem online na perspectiva da complexidade. 2007. Tese (Doutorado em Estudos Linguísticos) - Faculdade de Letras, Universidade Federal de Minas Gerais, Belo Horizonte, 2007.

FRANCO, Claudio. P. Por uma abordagem complexa de leitura. In: TAVARES, Kátia; BECHER, Siilvia; FRANCO, Claudio. (Orgs.). Ensino de leitura: fundamentos, práticas e reflexões para professores da era digital. Rio de Janeiro: Faculdade de Letras da UFRJ, 2011, p. 26-48. Disponível em: <http://www.claudiofranco.com.br/textos/franco_ebook_leitura.pdf >. Acesso em:3 nov. 2012.

- Autonomia na aprendizagem de inglês: um estudo com nativos digitais sob as lentes do caos e da complexidade.2013. Tese (Doutorado em Estudos Linguísticos) - Faculdade de Letras, Universidade Federal de Minas Gerais,Belo Horizonte, 2013.

JOHNSON, Neil. Simply complexity: a clear guide to complexity theory. Oxford: Oneworld, 2007. 
JOSLYN, Cliff; ROCHA, LuisM. Towards semiotic agent-based models of socio-technical organisations. In: AI, SIMULATION AND PLANNING (AIS 2000) CONFERENCE, Tucson, Arizona, USA, 2000, p.70-79.

LARSEN-FREEMAN, Diane. Chaos/complexity science and second language acquisition. Applied Linguistics. Oxford: Oxford University Press, v. 18, n. 2, p.141-165, 1997.

An attitude of inquiry: TESOL as science. The Journal of the Imagination in Language Learning, n. 5, p. 18-21, 2000.

- Language acquisition and language use from a chaos/complexity theory perspective. In: KRAMSCH, Claire. (Ed.) Language acquisition and language socialization: ecological perspectives, 2002. p. 88-95.

- The emergence of complexity, fluency, and accuracy in the oral and written production of five Chinese learners of English. Applied Linguistics. Oxford: Oxford University Press, v. 27, n. 4, p.590-619, 2006.

Adjusting expectations: the Study of complexity, accuracy, and fluency in second language acquisition. Applied Linguistics. Oxford: Oxford University Press, v. 30, n. 4, p.579-589, 2009.

LARSEN-FREEMAN, Diane; CAMERON, Lynne. Complex systems and Applied Linguistics.Oxford: Oxford University Press, 2008.

LEFFA, Vilson J. Transdisciplinaridade no ensino de línguas: a perspectiva das teorias da complexidade. Revista Brasileira de Linguística Aplicada, v. 6, n. 1, p. 27-49, 2006. Disponível em: <http://www.letras.ufmg.br/rbla/2006_1/02VilsonLeffa.pdf> Acesso em:2 abr. 2012.

Se mudo o mundo muda: ensino de línguas sob a perspectiva do emergentismo. Calidoscópio, v. 7, n. 1, p. 24-29, jan./abr. 2009. Disponível

em: 
<http://www.leffa.pro.br/textos/trabalhos/leffa_emergentismo.pdf> Acesso em:5 abr. 2012.

MARTINS, Antônio C. S. A emergência de eventos complexos em aulas on-line e face-a-face: uma abordagem ecológica. 2008. Tese (Doutorado em Estudos Linguísticos) - Faculdade de Letras, Universidade Federal de Minas Gerais, Belo Horizonte, 2008.

NEW ENGLAND COMPLEX SYSTEMS INSTITUTE. About complex systems. [s.d.]. Disponível em: <http://necsi.edu/guide/study.html>. Acesso em: 20 nov. 2012.

PAIVA, Vera L. M. O. Caleidoscópio: fractais de uma oficina de ensino aprendizagem. Memorial apresentado para concurso de Professor Titular na Faculdade de Letras da Universidade Federal de Minas Gerais, 2002. Disponível em: <http://www.veramenezes.com/memorial.htm>. Acesso em:3 abr. de 2012.

Modelo fractal de aquisição de línguas In: BRUNO, FátimaC. (Org.) Reflexão e prática em ensino/aprendizagem de língua estrangeira. São Paulo: Clara Luz, 2005a. p. 23-36. Disponível em: <http://www.veramenezes.com/modelo.htm>. Acesso em 3 abr. 2012.

Autonomia e complexidade: uma análise de narrativas de aprendizagem. In: FREIRE, Maximina. M; VIEIRA-ABRAHÃO, Maria H..; BARCELOS, Ana M. F. (Orgs.). Linguística Aplicada e contemporaneidade. Campinas; São Paulo: Pontes; ALAB, 2005 b. p.135-153.

em:

<http://www.veramenezes.com/autocomplex.htm>. Acesso em:3 abr. 2012.

Autonomia e complexidade. Linguagem \& Ensino, v. 9, n.1, p. 77-127, $2006 . \quad$ Disponível em: <http://rle.ucpel.tche.br/php/edicoes/v9n1/vera_paiva.pdf>. Acesso Em:2 nov. 2012. 
Aquisição e complexidade em narrativas multimídia de aprendizagem. Revista Brasileira de Linguística Aplicada, v. 8, n. 2. p. 321-339, 2008. Disponível em: <http://www.letras.ufmg.br/rbla/2008_2/03-Vera-Menezes.pdf>. Acesso em:3 abr. 2012.

- Caos, complexidade e aquisição de segunda língua. In: PAIVA, Vera L.M.O.; NASCIMENTO, Milton. (Org.). Sistemas adaptativos complexos: lingua(gem) e aprendizagem. Belo Horizonte: Faculdade de Letras da UFMG, 2009.p. 187-203.

Identity, motivation, and autonomy from the perspective of complex dynamical systems. In: MURRAY, Garold; GAO, Xuesong; LAMB, Terry. Identity, motivation and autonomy in language learning. Bristol, Buffalo, Toronto: MultilingualMatters, 2011. Disponível em: < http://www.veramenezes.com/identity.pdf >. Acesso em:2 nov. 2012.

PARREIRAS, Vicente A. A sala de aula digital sob a perspectiva dos sistemas complexos: uma abordagem qualitativa. 2005. Tese (Doutorado em Estudos Linguísticos) - Faculdade de Letras, Universidade Federal de Minas Gerais, Belo Horizonte, 2005.

RICHARDSON, Kurt A.; CILLIERS, Paul; LISSACK, Michael. Complexity science: A "gray" science for the "stuff in between". Emergence, v. 3, n. 2, p. 6-18, 2001. Disponível em: <http://wikinator.com/files/Richardson01.pdf〉. Acesso em:7 abr. 2012.

SADE, Liliane. A. R. Comunidades,identidade e aprendizagem de inglês sob a ótica do caos e dos sistemas complexos. 2009. Tese (Doutorado em Estudos Linguísticos) - Faculdade de Letras, Universidade Federal de Minas Gerais, Belo Horizonte, 2009.

SILVA, Valdir. A dinâmica caleidoscópica do processo de aprendizagem colaborativa no contexto virtual: Um estudo na perspectiva da complexidade/caos. Tese (Doutorado em Estudos Linguísticos) - Faculdade de Letras, Universidade Federal de Minas Gerais, Belo Horizonte, 2008. 
SILVA, Luciana O. Estágio supervisionado com uso de ambientes virtuais: possibilidades colaborativas. Tese (Doutorado em Estudos Linguísticos) - Faculdade de Letras, Universidade Federal de Minas Gerais, Belo Horizonte, 2013.

SOUZA, Valeska. Dinamicidade e adaptabilidade em comunidades virtuais de aprendizagem:uma textografia à luz do paradigma da complexidade. Tese (Doutorado em Estudos Linguísticos)- Faculdade de Letras, Universidade Federal de Minas Gerais, Belo Horizonte, 2011.

TEIXEIRA, Adriana. G. D. Difusão tecnológica no ensino de línguas: o uso de computadores portáteis nas aulas de língua portuguesa sob a ótica da complexidade. Tese (Doutorado em Estudos Linguísticos)Faculdade de Letras, Universidade Federal de Minas Gerais, Belo Horizonte, 2012.

VETROMILLE-CASTRO, Rafael. A interação social e o benefício recíproco como elementos constituintes de um sistema complexo em ambientes virtuais de aprendizagem para professores de línguas. Tese (Doutorado em Informática na Educação) - Faculdade de Educação, Universidade Federal do Rio Grande do Sul, Porto Alegre, 2007.

WALDROP, Mitchell. Complexity: the emerging science at the edge of order and chaos. New York: Simon \& Schuster, 1992.

Submetido em: $28 / 11 / 12$

Aceito em: 04/04/14

Title: A little about complexity in Applied Linguistics 